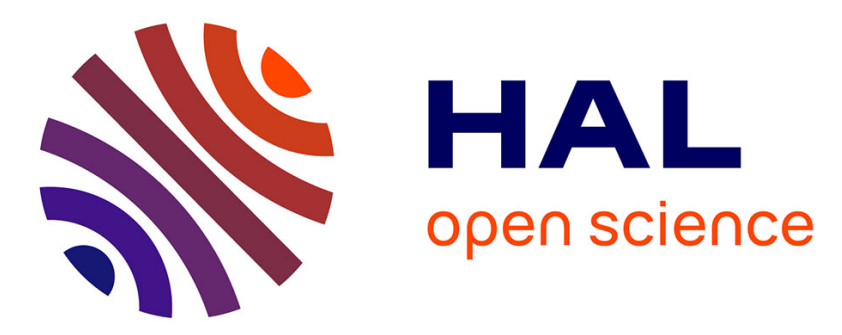

\title{
Pattern induced ordering of semiconducting graphene ribbons grown from nitrogen-seeded $\mathrm{SiC}$
}

Florence Ying Wang, G Liu, M. S. Nevius, Claire Mathieu, N Barrett, A. Sala, T. O. Mentes, Andrea Locatelli, P.I. Cohen, S. Rothwell, et al.

\section{- To cite this version:}

Florence Ying Wang, G Liu, M. S. Nevius, Claire Mathieu, N Barrett, et al.. Pattern induced ordering of semiconducting graphene ribbons grown from nitrogen-seeded SiC. Carbon, 2015, 82, pp.360-367. 10.1016/j.carbon.2014.10.081 . cea-01349660

\section{HAL Id: cea-01349660 https: / hal-cea.archives-ouvertes.fr/cea-01349660}

Submitted on 28 Jul 2016

HAL is a multi-disciplinary open access archive for the deposit and dissemination of scientific research documents, whether they are published or not. The documents may come from teaching and research institutions in France or abroad, or from public or private research centers.
L'archive ouverte pluridisciplinaire HAL, est destinée au dépôt et à la diffusion de documents scientifiques de niveau recherche, publiés ou non, émanant des établissements d'enseignement et de recherche français ou étrangers, des laboratoires publics ou privés. 


\title{
Pattern induced ordering of semiconducting graphene ribbons grown from nitrogen-seeded SiC
}

\author{
F. Wang ${ }^{a}$, G. Liu ${ }^{b}$, S. Rothwell ${ }^{c}$, M.S. Nevius ${ }^{a}$, C. Mathieu ${ }^{d}$, N. Barrett ${ }^{d}$, A. Sala ${ }^{e}$, \\ T.O. Menteş ${ }^{e}$, A. Locatelli ${ }^{e}$, P.I. Cohen ${ }^{c}$, L.C. Feldman ${ }^{b}$, E.H. Conrad ${ }^{a, *}$ \\ a School of Physics, The Georgia Institute of Technology, Atlanta, GA 30332-0430, USA \\ b Institute for Advanced Materials Devices and Nanotechnology, Rutgers University, Piscataway, NJ 08854, USA \\ c Department of Electrical and Computer Engineering, University of Minnesota, Minneapolis, MN 55455, USA \\ ${ }^{d}$ RAMIS/SPCSI/LENSIS, F-91191 Gif-sur-Yvette, France \\ e Elettra-Sincrotrone Trieste S.C.p.A., Basovizza, Trieste, Italy
}

\section{A R T I C L E I N F O}

Article history:

Received 30 June 2014

Accepted 24 October 2014

Available online 3 November 2014

\begin{abstract}
A B S T R A C T
A wide band gap semiconducting form of graphene can be produced by growing a buckled form of graphene from a $\mathrm{SiC}(000 \overline{1})$ surface randomly seeded with nitrogen. In this work, we show that the disorder observed in this form of graphene can be substantially reduced by pre-patterning the nitrogen seeded SiC surface into trenches. The result of the patterning is highly improved film thickness variations, orientational epitaxy, domain size, and electronic structure. The ordering induced by this patterned growth offers a way to take advantage of the extremely high mobilities and switching speeds in C-face graphene devices while having the thickness uniformity and fabrication scalability normally only achievable for graphene grown on the $\mathrm{SiC}(0001)$ Si-face.
\end{abstract}

(c) 2014 Elsevier Ltd. All rights reserved.

\section{Introduction}

Graphene grown from the $\operatorname{SiC}(000 \overline{1})$ (C-face) has exceptional mobilities because of its rotational stacking [1,2]. While controlling C-face graphene's thickness has been a problem, its potential use as an electronic material has made continuous progress. It has recently been used in a hybrid SiC-graphene Schottky barrier transistor with exceptional on/off ratios $\left(10^{6}\right)$ and relatively high channel mobility [3]. Furthermore, FETs built from monolayer $\mathrm{C}$-face graphene have shown the highest operating frequencies of any graphene-based devices [4]. New experiments on C-face SiC have recently shown that graphene grown from a C-face surface seeded with nitrogen (N-Gr) produces a large band gap form of graphene [5]. The nitrogen induced gap is thought to be a result of a strain gradient caused by a graphene-nitrogen-SiC bond that forces the graphene to buckle [6,7]. If strain is critical to the electronic structure of $\mathrm{N}-\mathrm{Gr}$, controlling the strain gradient will be required to develop this system as a new electronic material. One way to control the strain is to confine the N-Gr into small areas on a scale similar to the buckling. For example, by patterning the nitrogen-seeded layer prior to graphene growth, one can change the shape of the $\mathrm{N}-\mathrm{Gr}$ areas and control its strain dimensionality. Patterning also induces step edges that can act as heterogeneous desorption sites that allow Si to diffuse out of the graphene-SiC interface. Random Si desorption sites are known to be responsible for the wide thickness variations in C-face graphene films [8]. If the distance between edge sites can be patterned with dimensions similar to the surface Si diffusion length, a more spatially uniform graphene growth rate can be achieved.

As a first step to improving the order of $\mathrm{N}-\mathrm{Gr}$ films, we show that graphene growth from pre-patterned ribbons, formed by etching into a nitrogen seeded $\mathrm{SiC}(000 \overline{1})$ surface,

\footnotetext{
* Corresponding author. 
leads to three key improvements in the graphene film: (i) layer thickness control, (ii) improved rotational ordering, and (iii) a spatially uniform band structure. While the ribbon widths used in this study are too large to effect strain gradients in this system $(400 \mathrm{~nm} \leqslant w \leqslant 500 \mathrm{~nm})$, the distance between the step edges defining the ribbons is on the order of the distance between random vertical tubes that normally nucleate in C-face graphene films. We show that the ribbon structure inhibits the formation of the random vertical tubes with the result that graphene thickness uniformity is dramatically improved, presumably due to spatially controlled Si sublimation at the patterned step edges. Using a combination of Low Energy Electron Microscopy (LEEM), X-ray Photoelectron Microscopy (XPEEM) and micro-Angle Resolved Photoelectron Spectroscopy ( $\mu$-ARPES), we characterize both the topographical structure and band structure of $\mathrm{N}-\mathrm{Gr}$ ribbon arrays. We show that the graphene's structural properties are substantially improved within the confined ribbons when compared to 2D graphene. In fact, the thickness uniformity is improved to the point of single layer control. The results presented in this study are not only the first to use patterned ribbons to control growth, they are the only successful attempt to improve C-face graphene uniformity. The improved structural order is reflected in the electronic structure of the N-Gr ribbons, allowing more detailed measurements of the electronic properties of these films.

\section{N-Gr ribbon growth}

To produce this semiconducting form of graphene, we form $\sim 0.3 \mathrm{ML}\left(\sim 3.8 \times 10^{14} \mathrm{~cm}^{-2}\right)$ of nitrogen at a $\mathrm{SiC}(000 \overline{1}) / \mathrm{SiO}_{2}$ interface by high temperature growth in an atmospheric pressure NO gas [see Section 6]. The oxide is then removed leaving a bare $\mathrm{SiC}$ substrate seeded with nitrogen. The nitrogen produced this way is stable to high temperature $\left(>1300^{\circ} \mathrm{C}\right)$. This nitrogen-seeded surface is then patterned using a reactive ion etch (RIE) to remove nitrogen in stripes (referred to as trenches). We studied both $500 \mathrm{~nm}$ and $400 \mathrm{~nm}$ wide ribbons. The etch depth of a trench is $\sim 1 \mathrm{~nm}$ in these experiments. After patterning, the sample is heated in a closed RF induction furnace using the Confinement Controlled Sublimation method to form graphene [9]. A stable nitrogen coverage of $0.2 \mathrm{ML}$ is maintained after growth. No nitrogen is incorporated into the graphene lattice. Instead, the nitrogen remains between the graphene and the SiC surface as schematically illustrated in Fig. 1(a) [5]. It is known from previous studies that the nitrogen bonding between the first graphene layer and the $\mathrm{SiC}$ causes the graphene to buckle as shown in Fig. 1(a). As we'll show, most of the nitrogen remains on top of the ribbons as shown schematically in Fig. 1(b).

Fig. 1(c) shows a $10 \mu \mathrm{m}$ field of view (FOV) LEEM image of the $\mathrm{N}-\mathrm{Gr}$ array grown from a patterned nitrogen-seeded SiC surface. The $\mathrm{N}-\mathrm{Gr}$ ribbons are shown adjacent to a large unpatterned two-dimensional (2D) graphene area. The trenches appear dark in this image. Fig. 1(c) shows that the boundary of the shallow etched trenches remain sharp after graphene growth. It is important to note that the LEEM contrast is not due to the height differences of trenches and ribbons, but is instead due to a graphene thickness change at the boundary [see below]. While we cannot tell if the initial $1 \mathrm{~nm}$ steps remain after the $\mathrm{SiC}$ is consumed during graphene growth, Fig. 1(c) clearly demonstrates that the ribbon pattern remains intact.

\section{3. $\mathrm{N}-\mathrm{Gr}$ ribbon structure}

The first key observation about growth on patterned $\mathrm{N}-\mathrm{Gr}$ substrates is that the graphene thickness is more uniform on the ribbon area than on the 2D graphene areas of the same $(000 \overline{1})$ surface. In fact, the uniformity becomes similar to that achievable for graphene grown on the $\mathrm{SiC}(0001)$ (Si-face) [10]. Fig. 1(c) shows a LEEM image of both a ribbon patterned area and a standard 2D N-Gr film. While there are large contrast variations in the 2D N-Gr graphene regions, the contrast along the $\mathrm{N}-\mathrm{Gr}$ ribbons is very uniform. The contrast variations on the $2 \mathrm{D}$ area are due to graphene thickness changes typical in C-face graphene growth [11]. The extent of the thickness variation can be measured using LEEM reflectivity. The oscillations in LEEM reflectivity are due to the interference of the incoming electrons with the electrons that are reflected from the SiC-graphene interface [for details on LEEM reflectivity from graphene, see Ref. [12]]. The number of graphene layers is simply determined by counting the number of minima in a LEEM reflectivity spectrum between 0 and $10 \mathrm{eV}$ [12]. In the 2D area [see Fig. 1(d)], the number of minima in the LEEM reflectivity spectra show that the graphene thickness varies from 2 to 5 layers (a variance of $\sim 40 \%$ about the 3.5 layer average), typical of C-face graphene [11]. In contrast, Fig. 1(e) shows that the graphene on top of the nitrogen-seeded ribbons has a smaller thickness variation, varying by only one layer ( $\sim 9 \%)$ over the entire $10 \mu \mathrm{m}$ FOV. Note that the graphene is thicker $(\langle N\rangle \sim 5.5$ layers) on the ribbons compared to the 2D area. A comparison of the distribution of layer thickness for the ribbons and the 2D area is summarized in the thickness histogram in Fig. 1(f). The observed film uniformity on the ribbons represents a substantial advance in C-face graphene growth.

As determined from the LEEM reflectivity oscillations, the graphene thickness within the trenches is considerably less than that of the ribbons ( $\sim 2$-layers). The thickness variation between trench and ribbon areas is also confirmed by the laterally-resolved C1s photoemission spectra. Fig. 2(a) shows the $\mathrm{C} 1 \mathrm{~s}$ spectrum from both the $\mathrm{N}-\mathrm{Gr}$ ribbons and the trenches. The C1s spectrum in the trench region is typical of a thin C-face graphene film, showing a graphene peak and a relatively large $\mathrm{SiC}$ C1s peak [13]. On the ribbons, the SiC $\mathrm{C} 1$ s peak is smaller because the SiC C1s photoelectrons are attenuated as they pass through the thicker graphene film on the ribbon area. The difference in the graphene thickness is more evident in the XPEEM contrast image using the graphene C1s component [see Fig. 2(b)]. The N-Gr ribbons appear bright compared to the trench area because of the thicker $\mathrm{N}-\mathrm{Gr}$ film. The reduced growth in the trenches is most likely due to the RIE sputter damage induced in the SiC trenches during patterning. A similar effect is also observed on (0001)(Si-Face) graphene growth [14].

Both the thicker growth and the better thickness uniformity on the ribbons are consistent with an earlier model 


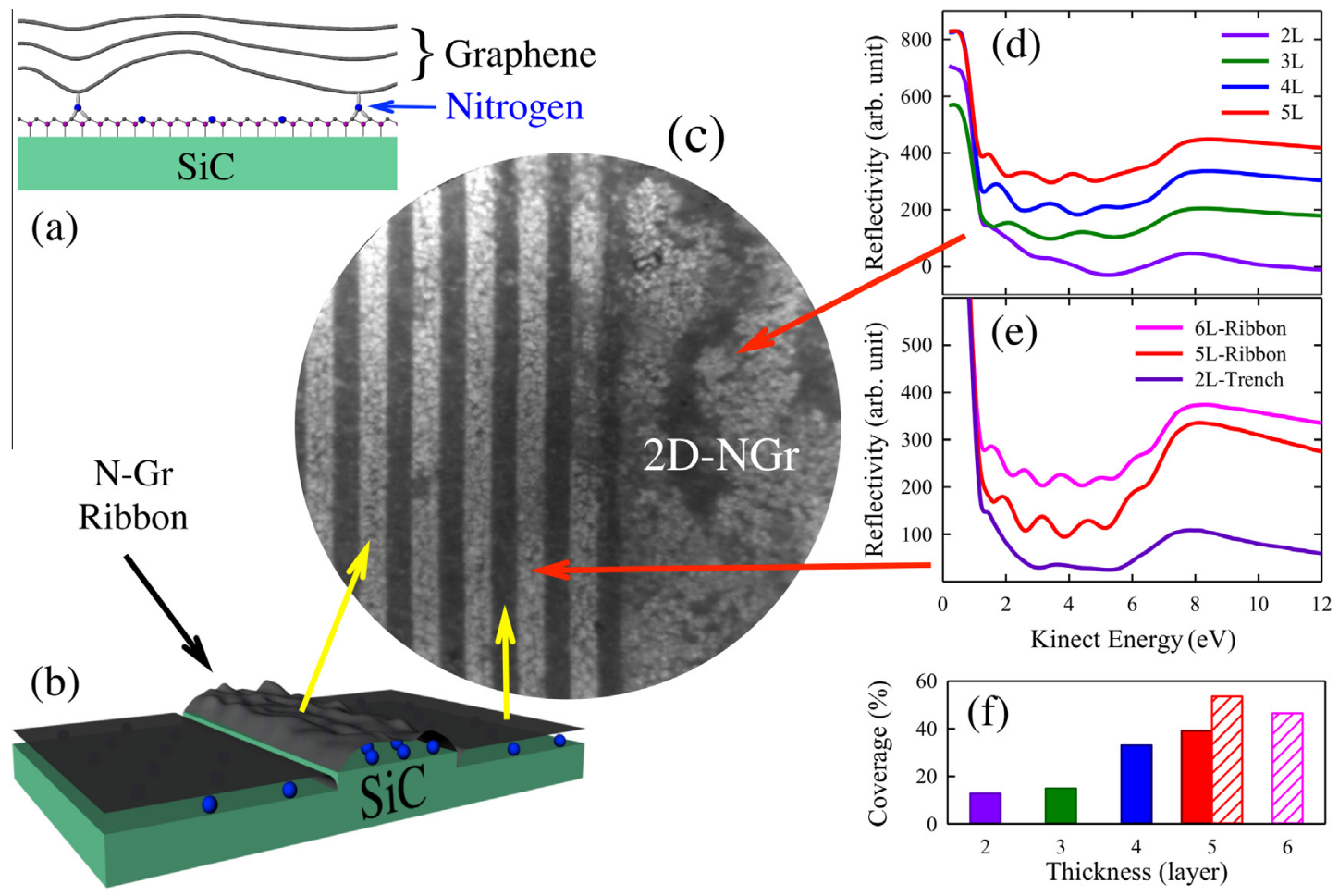

Fig. 1 - (a) Schematic model of the buckled N-Gr caused by nitrogen pinning of the first graphene layer to the SiC. (b) Schematic of the $\mathrm{N}-\mathrm{Gr}$ ribbons showing highly strained graphene above a nitrogen interface layer on the SiC. Nitrogen is shown in blue. (c) $10 \mu \mathrm{m}$ FOV LEEM image of a N-Gr ribbon array next to a $2 \mathrm{D} \mathrm{N}-\mathrm{Gr}$ area $(E=8.6 \mathrm{eV})$. The ribbon width is $w=500 \mathrm{~nm}$ and the pitch is $p=1 \mu \mathrm{m}$. (d) LEEM reflectivity spectrum from different regions in the 2D area of the sample with different numbers of graphene layers. The curves are shifted vertically for clarity. (e) LEEM reflectivity curves from the N-Gr ribbons and the trenches between ribbons. (f) A histogram of the relative coverage of different graphene thicknesses in the 2D area (solid bars) and in the ribbons (hatched bars). (A color version of this figure can be viewed online.)

proposed to explain the difference in graphene grown on (0001) (Si-face) and (0001) (C-face) surfaces [8]. In normal $\mathrm{C}$-face growth the local $\mathrm{Si}$ evaporation rate is determined by vertical tubes in the graphene film that extend from the SiC to the surface. The tubes are randomly dispersed across the $(000 \overline{1})$ surface. The tubes nucleate more readily on the $\mathrm{C}$-face when the interfacial $\mathrm{Si}$ concentration becomes large after the first few graphene layers form. In fact, this property has been used to grow vertical carbon nanotubes from C-face SiC [15]. Si can be easily transported away from the graphene-SiC interface where the vertical tubes form, causing the graphene to grow faster (i.e., thicker) in these areas. The result is a wide range of thickness variations across the surface due to the spatially random formation of these tubes. On the Si-face, these tubes do not form easily and the graphene film thickness is stunted to only a few layers as $\mathrm{Si}$ is trapped at the graphene-SiC interface. We suggest that the patterned step edges provide a similar but spatially controlled site for $\mathrm{Si}$ evaporation. Based on XPEEM and LEEM images, a typical distance from a tube to a boundary between different graphene thickness regions on the $\mathrm{C}$-face is a few microns [8]. The $500 \mathrm{~nm}$ width of the ribbons in these experiments means that Si diffusion to the edges will be sufficiently shorter than the known tube separation so that tube formation no longer determines the graphene thickness on the ribbon area. Therefore, unlike the 2D area, the interfacial Si concentration on the ribbons is both low and uniform leading to thicker graphene without the large thickness variations in the unpatterned areas.

We have also used XPEEM to measure the post-growth nitrogen distribution in the ribbon area. The nitrogen 1 s photoemission spectra for the trenches and ribbons are shown in Fig. 2(c). The distribution of the $\mathrm{N}$ 1s signal is displayed in the XPEEM image in Fig. 2(d). Although the trenches appear brighter in the N 1s image (more blue), the actual nitrogen distribution can only be determined by accounting for the attenuation of the $\mathrm{N}$ 1s signal through the graphene over-layer. This is done by comparing the attenuation of the SiC Si $2 p$ and the N 1s XPS signals through the known graphene thickness, as measured from the LEEM intensity versus voltage profiles in both the ribbons and trenches [see Section 6]. By this procedure, we find that the nitrogen in the trenches is half the nitrogen on the ribbons. Prior to these experiments, RIE test on macroscopic areas showed that a $1 \mathrm{~nm}$ etch was sufficient to remove the surface nitrogen below XPS detectable limits. Therefore, the presence of nitrogen in the trenches must be due to diffusion from the ribbon areas during graphene growth.

The second key observation from graphene grown on patterned substrates is the improved rotational epitaxy of the graphene in the ribbon area. Fig. 3(a) shows a $\mu$-LEED image from the 2D-graphene area. The LEED pattern shows the 

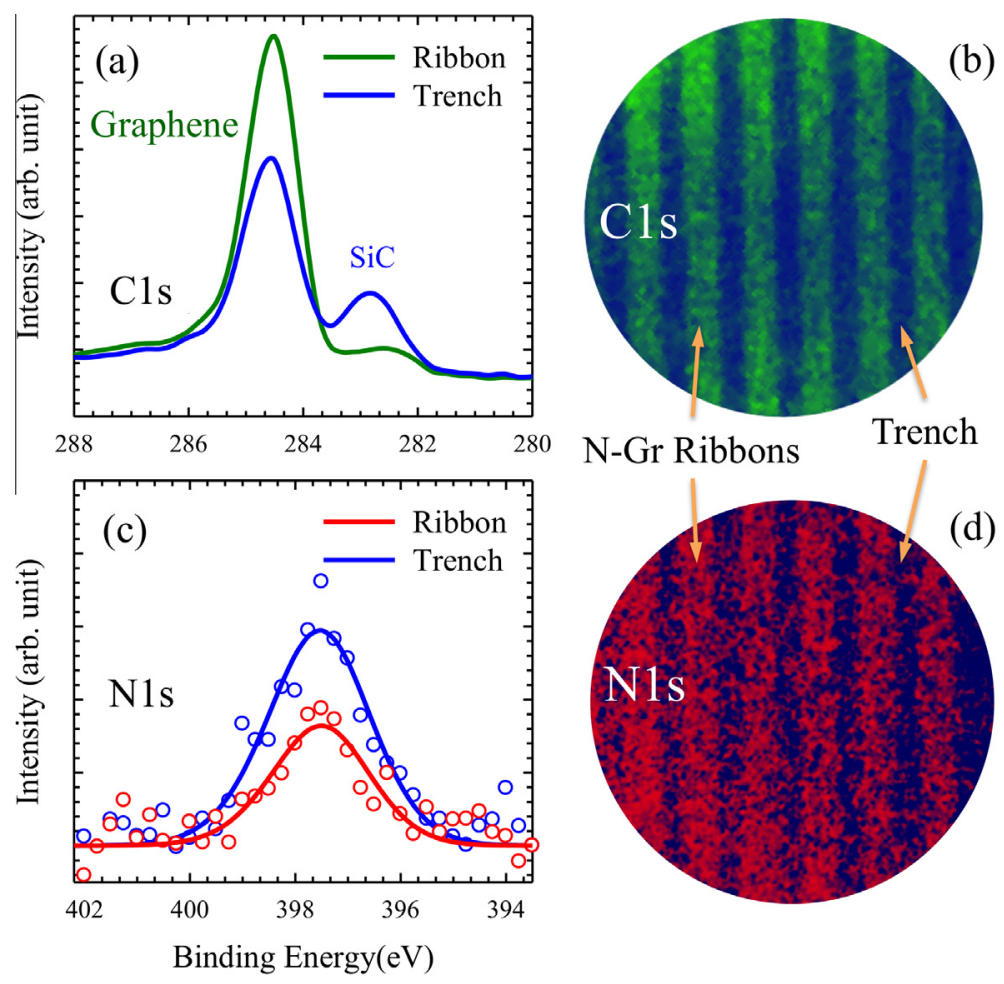

Fig. 2 - (a) C1s XPS spectra from the N-Gr ribbons and the trenches ( $h v=600 \mathrm{eV}$ ). The ribbon width is $w=400 \mathrm{~nm}$ and the pitch is $p=1 \mu \mathrm{m}$. (b) $6.5 \mu \mathrm{m}$ FOV XPEEM image using the graphene C1s peak at a binding energy (BE) of $B E=284.5 \mathrm{eV}$ ). Blue is from the trenches. (c) N 1s XPS spectra from N-Gr ribbons and etched trench bottoms ( $h v=600 \mathrm{eV}$ ). Background has been subtracted for both $\mathrm{N}$ 1s spectra. (d) $6.5 \mu \mathrm{m}$ Fov XPEEM image of the ribbons using the background subtracted $\mathrm{N} 1 \mathrm{~s}$ peak for contrast $(\mathrm{BE}=397.5 \mathrm{eV}$ ). Blue is the $\mathrm{N}$ 1s intensity in the trenches. (A color version of this figure can be viewed online.)

primary spots corresponding to graphene rotated $30^{\circ}$ relative to the $\operatorname{SiC}\langle 10 \overline{1} 0\rangle$ direction (brightest spots in the pattern) plus numerous diffraction patterns from rotationally (nonBernal) stacked graphene layers typical of C-face growth [16]. The additional spots are primarily from layers below the surface although some are from small rotated domain due to pleats or steps in the film [2,8]. A dark field (DF) LEEM image, using the graphene $(01)_{\mathrm{G}}$ spot for contrast, shows that the graphene's rotational orientation in the ribbons has become more homogeneous compared to the 2D area [see Fig. 3(b)]. Also note that the trench area has very little of the normal $30^{\circ}$ rotated graphene.

A more detailed look at the difference between graphene on the ribbon and trench areas can be made using dark field photoelectron microscopy (DF-PEEM). Fig. 3(c) shows a $\mu$-ARPES image of a constant energy cut through the graphene Brillouin zone (BZ) in the ribbon area. There are two rotated BZs: one from the primary $30^{\circ}$ graphene (the six brightest cones), and another from a graphene sheet rotated $\sim 0^{\circ}$ from the $\mathrm{SiC}\langle 10 \overline{1} 0\rangle$. We note that at the photon energies used in these experiments, the $\mu$-ARPES is sensitive to the top 3-layers because of inelastic electron attenuation. It is therefore difficult to determine if the $0^{\circ}$ rotated graphene is in the top graphene layer or in a rotated graphene sheet below the surface. A DF-PEEM image [17], using the $30^{\circ}$ cone for contrast, is shown in Fig. 3(d). The ribbons are bright in this image indicating that graphene in the ribbon area is predominately rotated $30^{\circ}$. In contrast, the trenches are dark indicating that most of the graphene in the trench area are rotated in other orientations. The observation that the ribbons and trenches have graphene both with different rotations (as determined by DF-PEEM and DF-LEEM) and different thicknesses (determined by LEEM reflectivity) confirm that the graphene is not continuous between the ribbons and trenches. As the schematic in Fig. 1(b) suggests, the graphene in the ribbon area must be isolated from the graphene in the trench area.

\section{Electronic structure of $\mathbf{N}-\mathrm{Gr}$ ribbons}

Fig. 4(a) and (c) show $E(k)$ cuts through the Dirac cone from the N-Gr ribbons and the un-patterned 2D N-Gr area, respectively. What is clear from Fig. 4(a) and (b) is that graphene in the $2 \mathrm{D}$ region has Dirac cones with much more intensity within the area bounded by the $\pi$-bands compared to the ribbons. This is seen more clearly in both energy distribution curves (EDC) and momentum distribution curves (MDC) through the cones. An EDC through the Dirac point of the ribbons area [see Fig. 4(b)] shows a peak in the density of states (DOS) at $0.5 \mathrm{eV}$ below $E_{F}$ signifying a band gap observed in nitrogen seeded graphene [5]. The DOS peak at the valence band maximum in Fig. 4(b) is well defined although broadened by the $0.3 \mathrm{eV}$ instrument resolution. The 2D area DOS in Fig. 4(d) shows no peak and a much larger background between the $\pi$-bands. It is important to note that a $2 \mu \mathrm{m}$ field limiting aperture was used in the $\mu$-ARPES. Therefore, in the ribbon area, the spectra are a composite of spectra from both 


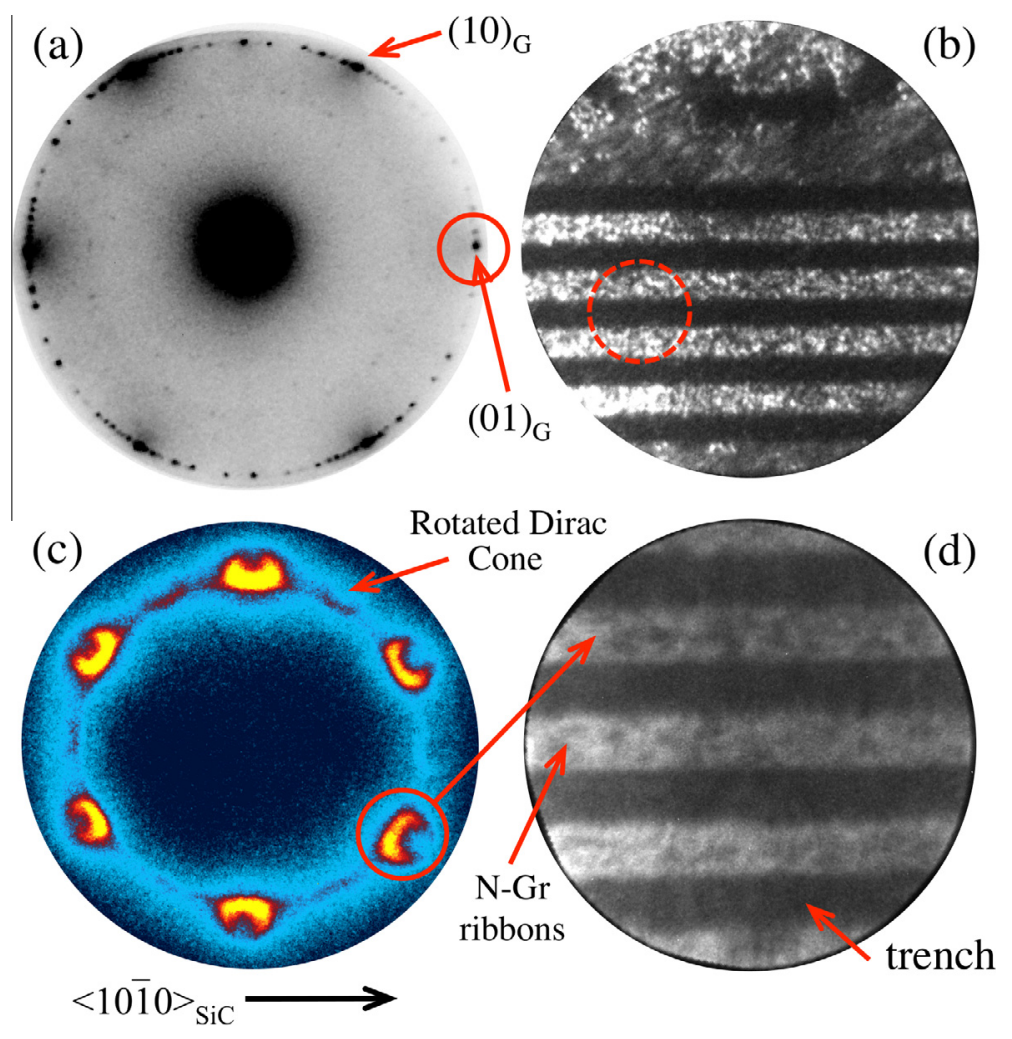

Fig. 3 - (a) $\mu$-LEED image from the $2 \mathrm{D}$ area showing that the primary graphene spots rotated $30^{\circ}$ from the SiC $\langle 10 \overline{10}\rangle$ direction. Other rotated domain spots are clearly visible. $E=40 \mathrm{eV}$. (b) DF-LEEM using the graphene $(01)_{\mathrm{G}}$ spot for contrast. $E=60 \mathrm{eV}$. $w=500 \mathrm{~nm}$. (c) Constant BE=-1.3 eV cut through the graphene BZ measured by $\mu$-ARPES of a 5-layer N-Gr ribbon area.

$h \omega=36 \mathrm{eV}$. The bright Dirac cones are the normal cones from the primary $30^{\circ}$ rotated graphene marked in (a). The faint cones are from graphene rotated at other commensurate angles. (d) DF-PEEM (BE $=-0.3 \mathrm{eV})$ of the N-Gr ribbons using the Dirac cone (red circle) in (c) and a $1.5 \mu \mathrm{m}$ contrast aperture [dashed circle in (b)]. (A color version of this figure can be viewed online.)

ribbons and trenches. However, within the region selected by the field limiting aperture, the ribbon area was twice that of trenches as marked by the dashed circle in Fig. 3(b). The intensity in the center of the cone, bounded by the $\pi$-bands in Fig. 4(a) and (c), is due to both the buckled graphene and the spatial disorder in the film. The N-Gr buckling contribution to the diffuse $E\left(k_{y}\right)$ spectrum is a result of the distribution of local surface normals[5] that cause electrons leaving different areas of the surface, with the same energy, to have different parallel momenta $\left(k_{x}, k_{y}\right)$. The result is that the analyzer simultaneously measures a wide set of slices through the Dirac cone, i.e., a set of constant $k_{x}$ conic sections through $E\left(k_{y}, k_{x}\right)$ for a broad range of different $k_{x}$ values [18]. Longrange order also influences the measured $E\left(k_{y}, k_{x}\right)$. Domain size is determined by intrinsic steps in the $\mathrm{SiC}$ or by steps caused by thickness variations in the film (i.e., thicker graphene areas have consumed more $\mathrm{SiC}$ and are therefore lower in height compared to thinner areas). Rotational boundaries can also be formed as graphene flows over a curved step. These finite size effects lead to a momentum broadening $\left(\Delta k_{x}, \Delta k_{y}\right)$ of the $\pi$-bands. MDC cuts through the cones [see Fig. 4(e)] shows that the $\pi$-bands are narrower in the ribbons compared to the $2 \mathrm{D}$ area. The $\Delta k_{y}$ width of the $2 \mathrm{D}$ area $\mathrm{N}-\mathrm{Gr}$ is twice as broad as the ribbons (the FWHM of the $2 \mathrm{D}$ and ribbons are $0.37 \AA^{-1}$ and $0.17 \AA^{-1}$, respectively). For comparison, the instrument resolution at this energy is $\Delta k_{\text {inst }} \sim 0.06 \AA^{-1}$. The significant reduction in the $\pi$-band broadening observed in the ribbons demonstrates that the ribbons geometry improves the long range order of the C-face film.

In addition to the $2 \mathrm{D} \mathrm{N}-\mathrm{Gr}$ spectra represented by Fig. 4(c) and (d), other parts of the 2D area have Dos curves similar to the ribbons but with a broader DOS peak [see Fig. 4(f)], more intensity between $\pi$-bands, and a larger $\Delta k_{y}$ broadening. A $\mu$-ARPES spectrum and its corresponding EDC from these "intermediate" areas are shown in Fig. 4(f) and $(g)$. Spectra of this type suggest that, within the 2D area, locally ordered areas typical of graphene on the ribbons coexist with more disordered areas characterized by spectra like those shown in Fig. 4(c) and (d). We demonstrate this by making a weighted sum of the two spectra in Fig. 4(b) and (d). The resultant composite spectra is shown in Fig. 4(g). The best fit is obtained for a ratio of 1:4 between the spectra from the disordered and ordered regions, respectively. Patterning the nitrogen seeded $\mathrm{SiC}$ before graphene growth has reduced the disordered $\mathrm{N}-\mathrm{Gr}$ regions that are prevalent throughout the $2 \mathrm{D}$ area. The increased order in these graphene ribbons will allow standard area-averaged ARPES (with better energy resolution) to be used in future studies design to investigate the role of strain in $\mathrm{N}-\mathrm{Gr}$ 's band structure. 

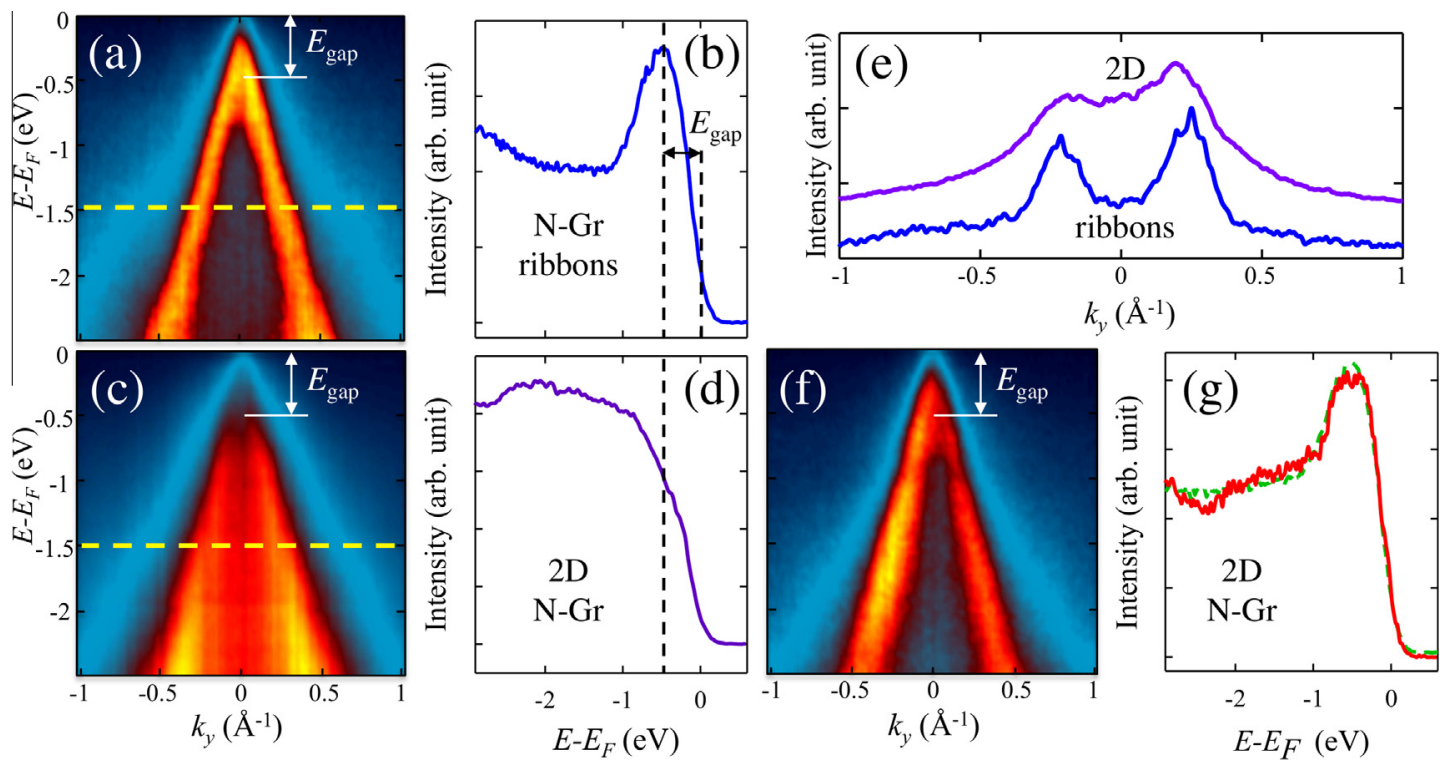

Fig. 4-E(k) slices through the Dirac cone (red circle in Fig. 3(c)) on (a) N-Gr ribbons and (c) on a representative area of the 2D NGr section. $k_{y}$ is perpendicular to the $\Gamma-K$ direction. (b) and (d) are corresponding EDCs through the Dirac point of the ribbon and 2D area, respectively. The valance band maximum in (b) is marked by the dashed vertical line. (e) MDC's through the Dirac cones in (a) and (c) at $E-E_{F}=-1.5 \mathrm{eV}$ [yellow dashed lines in (a) and (c)]. (f) $E(k)$ slices through a Dirac cone from a more ordered area of the 2D N-Gr section. (g) An EDC (red) through the Dirac point in (f) at $E-E_{F}=-1.5$ eV. A composite EDC (green dashed) is shown that is made from the EDC of the ribbon (b) and the EDC from the disordered 2D region in (d). (A color version of this figure can be viewed online.)

\section{Conclusions}

In this work, we have grown nitrogen-seeded graphene (N-Gr) from patterned stripes etched in the $\mathrm{SiC}(000 \overline{1})$ surface. The patterned growth produces a set of parallel nitrogen-seeded $\mathrm{SiC}$ stripes (400-500 nm wide). Graphene growth on these patterned surfaces leads to the formation of nitrogen-graphene ribbons with substantially improved structural and electronic properties. The thickness disorder normally observed in $\mathrm{C}$-face graphene is improved by a factor of four so that only monolayer variations are observed across at least $100 \mu \mathrm{m}^{2}$ area. Unfortunately, there is no theoretical guidance to understand the substantially improved thickness uniformity. Proposed growth models based on transmission electron microscopy studies of flat C-face graphene do not seem to apply to the ribbon geometry in this study [19]. We propose that the improved uniformity is a result of controlled heterogeneous edge sites in the patterned ribbons that allow Si to diffuse out of the graphene/SiC interface. The edge sites prevent $\mathrm{Si}$ buildup at the $\mathrm{SiC}$-graphene interface that normally causes the formation of vertical tube defects in the $\operatorname{SiC}(000 \overline{1})$ films [8], which act as Si vents that locally stimulate rapid graphene growth. In addition to the thickness control, rotational stacking order is also improved with the majority of the ribbon area having $\mathrm{N}-\mathrm{Gr}$ rotated $30^{\circ}$ relative to the $\mathrm{SiC}$ $\langle 10 \overline{1} 0\rangle$ direction. The improved thickness control and long range order of the $\mathrm{N}-\mathrm{Gr}$ ribbons result in a significant improvement in the uniformity of N-Gr's electronic band structure. The improved order will provide an experimental platform to investigate details of the band structure of $\mathrm{N}-\mathrm{Gr}$ films, including how its band gap depends on layer thickness, as well understanding how the large pseudo-magnetic fields, known to exist in these films, depend on strain confinement in sub-100 nm ribbons. While this work shows how to control the growth uniformity in $\mathrm{N}-\mathrm{Gr}$ ribbons, it also suggests that improvements in the film thickness of pristine graphene grown on $\mathrm{SiC}(000 \overline{1})$ surface can be made by patterning ribbons. This would offer a way to take advantage of the extremely high mobilities in C-face graphene and the thickness uniformity normally only achievable for graphene grown on the $\operatorname{SiC}(0001)$ (Si-face).

\section{Experimental method}

The substrates used in these studies were n-doped $n=2 \times 10^{18} \mathrm{~cm}^{-2} 4 \mathrm{H}$-SiC. To produce the initial nitrogen surface layers the $\mathrm{SiC}$ substrates were RCA cleaned. The samples were loaded into a $900^{\circ} \mathrm{C}$ furnace under a $500 \mathrm{sccm}$ Ar flow and heated to $1175^{\circ} \mathrm{C}$ over a $1 \mathrm{~h}$ ramp. The sample is then kept at $1175^{\circ} \mathrm{C}$ for $2 \mathrm{~h}$ (for a $0.3 \mathrm{ML}$ nitrogen coverage) with a $500 \mathrm{sccm}$ NO flow [20]. The sample is then cooled to $900^{\circ} \mathrm{C}$ under a $500 \mathrm{sccm}$ Ar flow and unloaded from furnace. Oxide grown through this anneal is removed by HF immediately before graphene growth. The trench arrays were prepared by first producing a negative ZEP mask by e-beam lithography. The patterned SiC substrate is then reactive ion etched with a $\mathrm{SF}_{6}-\mathrm{O}_{2}-$ Ar plasma to produce up to 1-2 $\mathrm{nm}$ deep trenches depending on etching time. After removing the mask material, the C-face nitrogen-seeded surfaces were then heated in a closed graphite crucible in an RF vacuum furnace to 
$1450^{\circ} \mathrm{C}$ to produce the graphene films [9]. This growth temperature is slightly higher than the desorption temperature of nitrogen as discussed in the supplement.

The nitrogen coverage, $N_{N}$, is estimated from the ratio of the $\mathrm{N} 1 \mathrm{~s}$ to $\mathrm{Si} 2 \mathrm{p}$ intensities $N_{\mathrm{N}}=\left(\mathrm{I}_{\mathrm{N}} / \mathrm{I}_{\mathrm{Si}}\right)\left(\sigma_{\mathrm{Si}} / \sigma_{\mathrm{N}}\right) n_{\mathrm{Si}} \lambda$, where $I_{N}$ and $I_{S i}$ are the $\mathrm{N} 1 \mathrm{~s}$ and $\mathrm{Si} 2 \mathrm{p}$ XPS intensities, $\sigma_{\mathrm{N}}$ and $\sigma_{\mathrm{Si}}$ are the photoionization cross sections of $\mathrm{N}$ and $\mathrm{Si}[21,22]$. $n_{\mathrm{Si}}=4.8 \times 10^{22} / \mathrm{cm}^{3}$ is atomic density of $\mathrm{Si}$ in $\mathrm{SiC}$. For this work, we use a mean free path in $\operatorname{SiC}$ of $\lambda=2.2 \mathrm{~nm}$ at $1486 \mathrm{eV}$.

The ratio of the nitrogen coverage between the ribbon area, $\theta_{r}$, and the trench area, $\theta_{t}$, is estimated from the ratio of the $\mathrm{N}$ 1s to $\mathrm{Si} 2 \mathrm{p}$ intensities in two areas:

$\frac{\theta_{r}}{\theta_{t}}=\frac{\left(I_{N} / I_{S i}\right)_{r}}{\left(I_{N} / I_{S i}\right)_{t}} \times \frac{e^{-d_{t}\left(1 / \lambda_{N}-1 / \lambda_{S i}\right)}}{e^{-d_{r}\left(1 / \lambda_{N}-1 / \lambda_{S i}\right)}}$,

where $\left(I_{N} / I_{S i}\right)_{r}$ and $\left(I_{N} / I_{S i}\right)_{t}$ are the ratio of the $N$ 1s and Si $2 p$ XPS intensities from ribbon and trench areas, respectively. $\lambda_{N}$ and $\lambda_{\mathrm{Si}}$ are the electron mean free paths in graphene from a nitrogen and silicon photoelectron, respectively. $d_{r}$ and $d_{t}$ are the graphene thickness in the ribbon and trench areas.

The photoemission microscopy measurements were carried out using the spectroscopic photoemission and low energy electron microscope (SPELEEM) at the Elettra Synchrotron Light Laboratory [23]. This instrument combines Low Energy Electron Microscopy [24] with energy- filtered X-ray photoemission microscopy [25]. In the SPELEEM, the electron kinetic energy is controlled by biasing the sample with a negative potential. This bias is referred to as start voltage, $V_{\text {start }}$. The kinetic energy of the electrons scattered (or emitted) by the sample is equal to $E_{\text {kin }}=V_{\text {start }}-\delta \mathrm{W}_{i-s}$, the latter being the difference in work function between the instrument and the specimen. The microscope lateral resolution approaches a few tens of nanometers; energy resolution is better than $0.3 \mathrm{eV}$. Along with imaging, the SPELEEM allows diffraction operation mode. Depending whether the beamline photons or low energy electrons are used as probe, $\mu$-ARPES or LEED measurements can be also carried out. The probed area, about $2 \mu \mathrm{m}$ in diameter, is chosen by inserting a field limiting or illumination aperture in the optical path of the instrument. Part of the ARPES measurements was made utilizing the DFPEEM method [17].

\section{Acknowledgments}

This research was supported by the NSF under Grant Nos. DMR-1206793, DMR-1206655, and DMR-1206256. Additional support is also acknowledged from the NSF DMR-1005880 and the W.M. Keck Foundation.

\section{R E F E R E N C E S}

[1] de Heer WA, Berger C, Wu X, First PN, Conrad EH, Li X, et al. Epitaxial graphene. Solid State Commun 2007;143:92.

[2] Hass J, de Heer WA, Conrad EH. The growth and morphology of epitaxial multilayer graphene. J Phys Condens Matter 2008;20:323202-12.

[3] Y. Hu, Production and properties of epitaxial graphene on carbon terminated face of hexagonal silicon carbide $[\mathrm{PhD}$ thesis]. Georgia Institute of Technology; 2013. http:// hdl.handle.net/1853/48705.

[4] Guo Z, Dong R, Chakraborty PS, Lourenco N, Palmer J, Hu Y, et al. Record maximum oscillation frequency in C-face epitaxial graphene transistors. Nano Lett 2013;13:942.

[5] Wang F, Liu G, Rothwell S, Nevius M, Tejeda A, Taleb-Ibrahimi A, et al. Wide-gap semiconducting graphene from nitrogenseeded SiC. Nano Lett 2013;13:4827.

[6] Low T, Guinea F. Strain-induced pseudomagnetic field for novel graphene electronics. Nano Lett 2010;10:3551.

[7] Low T, Guinea F, Katsnelson MI. Gaps tunable by electrostatic gates in strained graphene. Phys Rev B 2011;83:195436.

[8] Hicks J, Shepperd K, Wang F, Conrad EH. The structure of graphene grown on the $\mathrm{SiC}(000 \overline{1})$ surface. J Phys D Appl Phys 2012;45:154002.

[9] de Heer WA, Berger C, Ruan M, Sprinkle M, Li X, Hu Y, et al. Large area and structured epitaxial graphene produced by confinement controlled sublimation of silicon carbide. Proc Natl Acad Sci 2011;108:16900.

[10] Moon J-S, Curtis D, Hu M, Wong D, Campbell PM, Jernigan G, et al. Development toward wafer-scale graphene RF electronics. Topical meeting on silicon monolithic integrated circuits in RF systems (SiRF) 2010;1:11-3.

[11] Wang F, Shepperd K, Hicks J, Nevius MS, Tinkey H, Tejeda A, et al. Silicon intercalation into the graphene-SiC interface. Phys Rev B 2012;85:165449. 6.

[12] Hibino H, Kageshima H, Maeda F, Nagase M, Kobayashi Y, Yamaguchi H. Microscopic thickness determination of thin graphite films formed on $\mathrm{SiC}$ from quantized oscillation in reflectivity of low-energy electrons. Phys Rev B 2008;77:075413 (7).

[13] Emtsev KV, Speck F, Seyller Th, Ley L, Riley JD, et al. Interaction, and ordering of epitaxial graphene on $\mathrm{SiC}\{0001\}$ surfaces: a comparative photoelectron spectroscopy study. Phys Rev B 2008;77:155303.

[14] Nevius MS, Wang F, Mathieu C, Barrett N, Sala A, Menteş TO, et al. The bottom-up growth of edge specific graphene nanoribbons. Nano Lett 2014. http://dx.doi.org/10.1021/nl502942z. Article ASAP.

[15] Kusunoki M, Suzuki T, Kaneko K, Ito M. Formation of selfaligned carbon nanotube films by surface decomposition of silicon carbide. Philos Mag Lett 1999;79:153.

[16] Sprinkle M, Hicks J, Tejeda A, Taleb-Ibrahimi A, Le Fèvre P, Bertran F, et al. Multilayer epitaxial graphene grown on the $\mathrm{SiC}(000 \overline{1})$ surface; structure and electronic properties. J Phys D Appl Phys 2010;43:374006 (12pp).

[17] Menteş TO, Locatelli A. Angle-resolved X-ray photoemission electron microscopy. J Electron Spectrosc Relat Phenom 2012;185:32317329.

[18] Knox KR, Wang S, Morgante A, Cvetko D, Locatelli A, Menteş TO, et al. Spectromicroscopy of single and multilayer graphene supported by a weakly interacting substrate. Phys Rev B 2008;78:201408 (R).

[19] Norimatsu W, Kusunoki M. Growth of graphene from $\mathrm{SiC}\{0001\}$ surfaces and its mechanisms. Semicond Sci Technol 2014;29:064009.

[20] Liu G, Ahyi AC, Xu Y, Isaacs-Smith T, Sharma YK, Williams JR, et al. Enhanced inversion mobility on $4 \mathrm{H}-\mathrm{SiC}(1 \overline{1} \overline{2} 0)$ using phosphorus and nitrogen interface passivation. IEEE Electron Device Lett 2013;34:181.

[21] Yeh J-J, Lindau I. Atomic subshell photoionization cross sections and asymmetry parameters: $1<Z<103$. Atomic Data Nucl Data Tables 1985;32:1.

[22] J.-J. Yeh, Atomic calculations of photoionization cross sections and asymmetry parameters, Gordon and Breach, Langhorne, PA, 1993.

[23] Locatelli A, Aballe L, Menteş TO, Kiskinova M, Bauer E. Photoemission electron microscopy with chemical 
sensitivity: SPELEEM methods and applications. Surf Interface Anal 2006;38:1554171557.

[24] Bauer E. Low energy electron microscopy. Rep Prog Phys 1994;57:895.
[25] Schmidt T, Heun S, Slezak J, Diaz J, Prince KC, Lilienkamp G, et al. SPELEEM: combining LEEM and spectroscopic imaging. Surf Rev Lett 1998;5:1287171296. 